University of Nebraska - Lincoln

DigitalCommons@University of Nebraska - Lincoln

$12-17-1998$

Experimental observation of relativistic nonlinear Thomson scattering

Szu-yuan Chen

University of Michigan, Ann Arbor, Michigan

Anatoly Maksimchuk

University of Michigan, tolya@umich.edu

Donald Umstadter

University of Nebraska - Lincoln, donald.umstadter@unl.edu

Follow this and additional works at: https://digitalcommons.unl.edu/physicsumstadter

Part of the Physics Commons

Chen, Szu-yuan; Maksimchuk, Anatoly; and Umstadter, Donald, "Experimental observation of relativistic nonlinear Thomson scattering" (1998). Donald Umstadter Publications. 75.

https://digitalcommons.unl.edu/physicsumstadter/75

This Article is brought to you for free and open access by the Research Papers in Physics and Astronomy at DigitalCommons@University of Nebraska - Lincoln. It has been accepted for inclusion in Donald Umstadter Publications by an authorized administrator of DigitalCommons@University of Nebraska - Lincoln. 
Published in Nature 396 (December 17, 1998), pp. 653-655.

Copyright (C) 1998 Macmillan Publishers Ltd. Used by permission. http://www.nature.com

Submitted May 28, 1998; accepted September 23, 1998.

\title{
Experimental observation of relativistic nonlinear Thomson scattering
}

\author{
Szu-yuan Chen, Anatoly Maksimchuk, and Donald Umstadter \\ Center for Ultrafast Optical Science, University of Michigan, Ann Arbor, Michigan 48109, USA \\ Corresponding author: D. Umstadter
}

Classical Thomson scattering ${ }^{1}$ - the scattering of low-intensity light by electrons - is a linear process, in that it does not change the frequency of the radiation; moreover, the magnetic-field component of light is not involved. But if the light intensity is extremely high $\left(\sim 10^{18} \mathrm{Wcm}^{-2}\right)$, the electrons oscillate during the scattering process with velocities approaching the speed of light. In this relativistic regime, the effect of the magnetic and electric fields on the electron motion should become comparable, and the effective electron mass will increase. Consequently, electrons in such high fields are predicted to quiver nonlinearly, moving in figure-eight patterns rather than in straight lines. Scattered photons should therefore be radiated at harmonics of the frequency of the incident light ${ }^{2-8}$, with each harmonic having its own unique angular distribution. ${ }^{4-6}$ Ultrahigh-peak-power lasers ${ }^{9}$ offer a means of creating the huge photon densities required to study relativistic, or "nonlinear" (ref. 6), Thomson scattering. Here we use such an approach to obtain direct experimental confirmation of the theoretical predictions of relativistic Thomson scattering. In the future, it may be possible to achieve coherent ${ }^{10,11}$ generation of the harmonics, a process that could be potentially utilized for "table-top" X-ray sources.

We used a laser system that produces 400 -fs-duration laser pulses at $1.053-\mu \mathrm{m}$ wavelength with a maximum peak power of 4 $\times 10^{12} \mathrm{~W}(4 \mathrm{TW})$. The laser beam, $50 \mathrm{~mm}$ in diameter, was focused with an $f / 3.3$ parabolic mirror onto the front edge of a supersonic helium gas jet. The focal spot consisted of a gaussian spot with full-width at half-maximum (FWHM) of $7 \mu \mathrm{m}$ (containing $60 \%$ of the total energy) and a large $(>100 \mu \mathrm{m})$ dim spot. The helium gas was fully ionized by the foot of the laser pulse. A halfwave plate was used to rotate the axis of linear polarization of the laser beam in order to vary the azimuthal angle $(\varphi)$ of observation. We define $\theta$ $=0^{\circ}$ as along the direction opposite to that of the laser propagation, and $\varphi=0^{\circ}$ as along the axis of linear polarization. In a linearly polarized laser field, electrons move in a figure-eight trajectory lying in the plane defined by the axis of linear polarization and the direction of beam propagation.

Although the observation of harmonics in laser-plasma (or laser-electron-beam) interactions has been made by several groups, ${ }^{12-16}$ that alone is insufficient to identify unambiguously nonlinear Thomson scattering and its underlying dynamics. Several
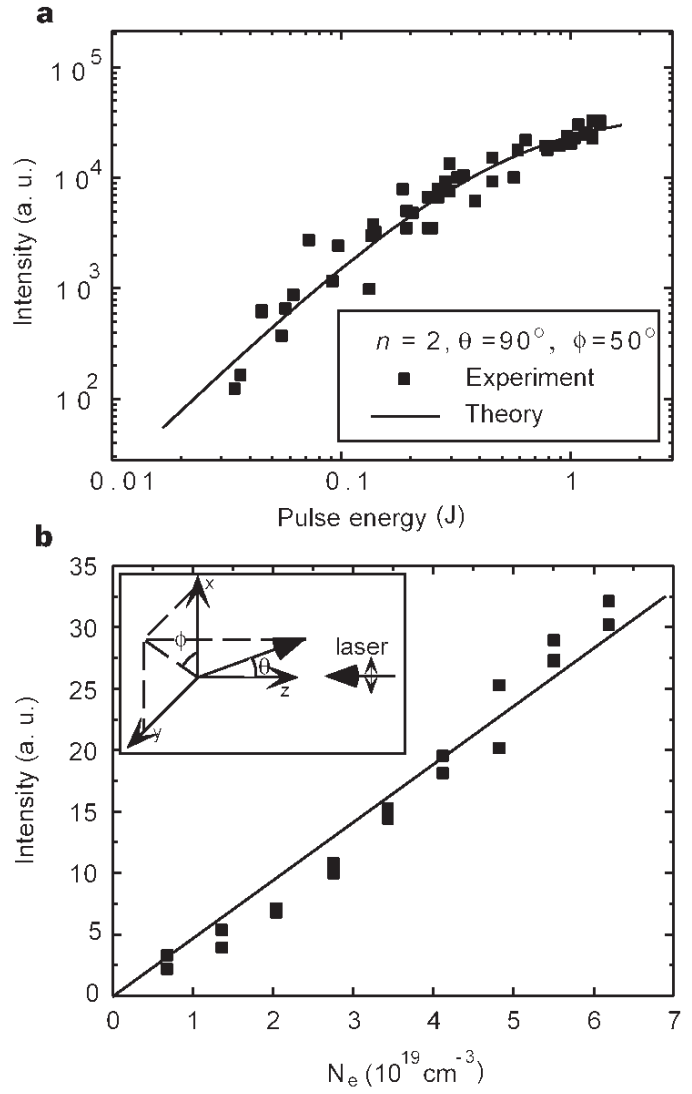

Figure 1 Dependence of the second-harmonic light on laser intensity and plasma density. Shown is the intensity of the second-harmonic light at $\theta=90^{\circ}, \varphi=50^{\circ}$ : a.) as a function of laser pulse energy at 6.2 $\times 10^{19} \mathrm{~cm}^{-3}$ electron density, and $\mathbf{b}$.) as a function of plasma electron density $\left(N_{\mathrm{e}}\right)$ at $0.8-\mathrm{J}$ laser pulse energy. (For 1-J laser pulse energy, the laser intensity is $4: 4 \times 10^{18} \mathrm{Wcm}^{-2}$ and $a_{0}$ (see text) is 1.88.) Each data point represents the result of a single laser shot. The theoretical predictions for zero drift velocity are plotted as solid lines, for comparison. The only fitting parameter is a constant for normalization in all figures. The laser pulse energy refers to the energy in the main focal spot. The inset shows the coordinate system used. 
other mechanisms might generate continuum or harmonics under our experimental conditions, and, therefore, need to be isolated and discriminated from the signal generated by nonlinear Thomson scattering: (1) continuum generated from self-phase-modulation of the laser beam in the gas, (2) harmonics generated from atomic nonlinear susceptibility of the gas or, especially, from the ionization process, ${ }^{17}$ (3) continuum generated either from (relativistic) self-phase-modulation of laser pulses in the plasma, or from electron-electron bremsstrahlung and electron-ion bremsstrahlung, and (4) harmonics generated from the interaction of laser pulses with a transverse electron-density gradient. ${ }^{14}$

The main focal spot of the laser pulse undergoes relativisticponderomotive self-channeling when high laser power and high gas density are used. ${ }^{18}$ (The ponderomotive force is generated by the gradient of the optical pressure of the laser beam). Side-imaging ( $\theta$ $=90^{\circ}$ ) of the first-harmonic light (at the laser frequency) from nonlinear Thomson scattering shows that the laser channel has a diameter of $<10 \mu \mathrm{m}$ FWHM. However, interferograms ${ }^{18}$ show that the diameter of the plasma column is about 100-200 $\mu \mathrm{m}$, which is created by the wings with intensities $>10^{15} \mathrm{Wcm}^{-2}$ (the ionization threshold). Therefore, the light generated from laser-gas interactions should be observed to originate from the entire region of plasma, rather than from the narrow laser channel. Results of

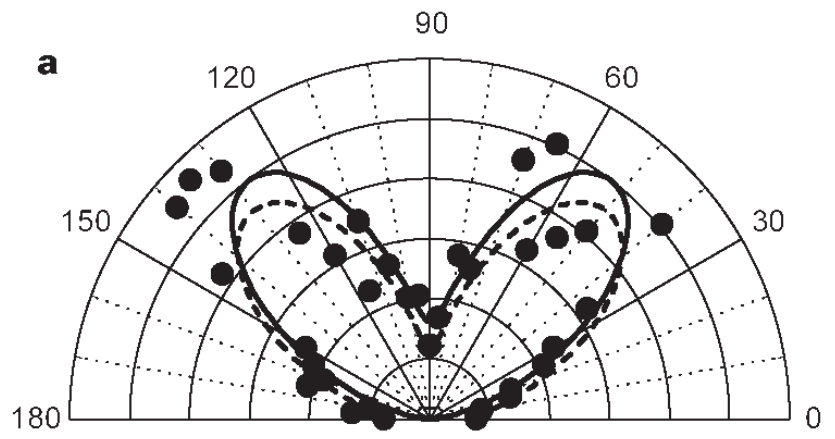

90

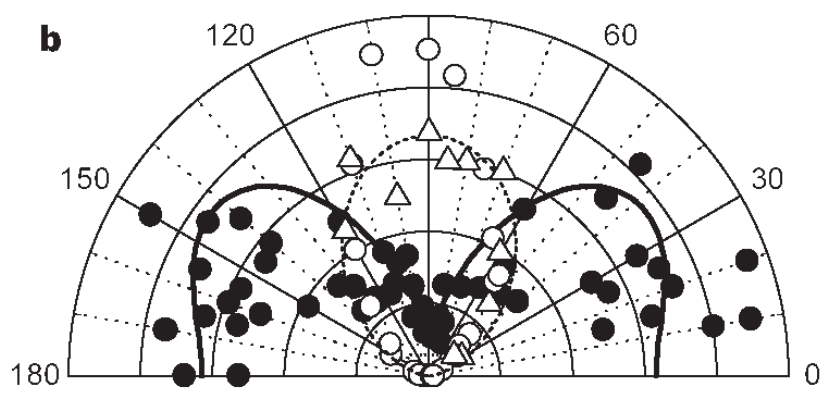

Figure 2 Angular pattern of the second-harmonic light. Shown are polar plots of the intensity of the second-harmonic light as a function of azimuthal angle $\left(\varphi\right.$, in degrees) for $0.8-\mathrm{J}$ pulse energy and $6.2 \times 10^{19}$ $\mathrm{cm}^{-3}$ electron density at $\theta=90^{\circ}$ (a) and $\theta=51^{\circ}$ (b). The intensity is in arbitrary units. Filled circles, experimental data; solid and dashed lines, theoretical results for zero and nonzero drift velocity $(v=0.2 c)$ in the laser propagation direction, respectively. Open circles and triangles, experimental data for the first-harmonic signal taken at two different runs under the same conditions; dotted line, the theoretical result. Its dipole radiation pattern (peaked at $\varphi=90^{\circ}$ ) confirms that there is no depolarization effect in the plasma and that the collective effect of plasmas on the angular pattern is not significant (at least outside a narrow cone along the axis of laser propagation). Although the data for $\varphi=180^{\circ}$ to $360^{\circ}$ are not plotted, they should be a mirror image of the data for $\varphi=0^{\circ}$ to $180^{\circ}$ because of the intrinsic symmetry of the laser field, as predicted theoretically. Such symmetry has been verified in the experiment. side-imaging $\left(\theta=90^{\circ}\right.$ and arbitrary $\left.\varphi\right)$ of the second and third harmonics using a matching interference filter (10-nm bandwidth) show that the signal is emitted only from the narrower laser channel. In addition, the images of the harmonics have spatial distributions similar to the images of the first-harmonic light, and their profiles vary in the same way as the laser power and gas density are changed. This rules out the possibility that the harmonic signal observed in the side images is a result of laser-gas interaction described in mechanisms (1) and (2) above.

According to theory, ${ }^{4-6}$ the harmonic signal generated from nonlinear Thomson scattering should have two important features. First, it is linearly proportional to the electron density because it is an incoherent single-electron process (the harmonics generated from a collection of electrons interfere with each other destructively, leaving only an incoherent signal, which is equal to the single-electron results multiplied by the total number of electrons which radiate). Second, it increases roughly as $I^{n}$, where $n$ is the harmonic number, and gradually saturates when $a_{0}$ is of the order of unity. ${ }^{6}$ Here $a_{0}=e E / m_{0} \omega_{0} c=8: 5 \times 10^{-10} \lambda I^{1 / 2}$ is the normalized vector potential, where $E$ is the amplitude of laser electric field, $I=c E^{2} / 8 \pi$ (in $\mathrm{Wcm}^{-2}$ ) is the laser intensity, $\lambda$ is the laser wavelength (in $\mu \mathrm{m}$ ), $e$ is the electron charge, $m_{0}$ is the electron mass, $\omega_{0}$ is the laser frequency, and $c$ is the speed of light. These are characteristically different from the behavior of any other mechanisms. For instance, bremsstrahlung radiation should be proportional to the square of gas density $\left(N_{\mathrm{e}} N_{\mathrm{e}}\right.$ or $\left.N_{\mathrm{e}} N_{\mathrm{i}}\right)$ where $N_{\mathrm{e}}$ is the electron density and $N_{\mathrm{i}}$ is the ion density. In the experiment reported here, the intensity of the harmonic signal was determined from the peak intensity or the average intensity of the images of harmonics, when it was plotted as a function of the observing angle, gas density and laser power. Both showed the same variations. Figure 1 shows the variation of the second-harmonic signal as a function of laser power and plasma (electron) density. The experimental results show a reasonable match to the theoretical predictions. The first and third harmonics show the same match with the theory.

Although the above two observations are consistent with nonlinear Thomson scattering as the source of the harmonic signal, observations of the unique angular patterns are necessary to prove that the detailed dynamics of nonlinear Thomson scattering are indeed the same as the theoretical prediction. Figure 2a shows the $\varphi$ dependence of the second-harmonic signal at $\theta=90^{\circ}$. The exper-

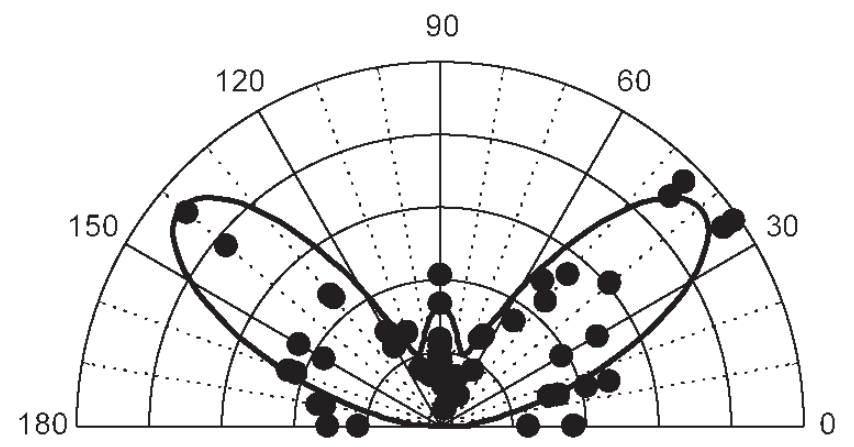

Figure 3 Angular pattern of the third-harmonic light. Shown is a polar plot of the intensity of the third-harmonic light as a function of azimuthal angle $\left(\varphi\right.$, in degrees) at $\theta=90^{\circ}$ for 0.8 -J pulse energy and 6.2 $\times 10^{19} \mathrm{~cm}^{-3}$ electron density. The intensity is in arbitrary units. Filled circles, experimental data; solid line, the theoretical result for zero drift velocity. The angular patterns of harmonics should not be sensitive to variation of laser intensity, as expected from theory and checked in the experiment. This is crucial to the success of our measurements because it alleviates the error caused by fluctuations of laser intensity. 
imental results match qualitatively the theoretical prediction, both having a quadrupole-type radiation pattern which is characteristically different from the dipole pattern for other mechanisms (1)(4), and linear Thomson scattering. Other measurements, such as the $\varphi$-dependence of the second-harmonic light at $\theta=51^{\circ}$ (an "antidipole" pattern; Figure 2b), and the $\varphi$-dependence of the third-harmonic light at $\theta=90^{\circ}$ (a "butterfly" pattern; Figure 3), were also made, all showing reasonable matches between the experimental data and the theoretical predictions. Such angular radiation patterns directly prove the electrons do indeed oscillate with a figure-eight trajectory in an intense (relativistic) laser field. The angular pattern of the first-harmonic light (linear component) of nonlinear Thomson scattering is also included in Figure $2 b$ for comparison.

The spectra of the second and the third harmonics each contain a peak at roughly the harmonic wavelength and a red-shifted broader peak, as shown in Figure 4. The red-shifted broader peaks are believed to be part of the harmonic spectra generated by nonlinear Thomson scattering, because they vary in amplitude proportionally with the corresponding unshifted harmonic signals when the gas density and laser power are changed. It was expected that the spectra of the harmonics should be broadened tremendously for electrons in a high-fluid-velocity plasma wave. ${ }^{5}$ A fast-phase-velocity electron plasma wave (with a maximum fluid velocity of $0.2 c$ ) excited by stimulated Raman forward scattering ${ }^{19}$ was observed in this experiment at the highest laser power and gas density. But the spectral distribution of the harmonics was not observed to change significantly with variation of gas density and laser power, whereas the plasma wave amplitude did change with these variations. This indicates that such spectral structure has nothing to do with the collective drift motion of electrons in the plasma waves.

Although the angular radiation patterns of the harmonics could also be affected by such a $0.2 c$ fluid-velocity oscillation, the changes are not significant enough (compare the solid and dashed lines in Figure 2a) to be identified from the experimental data. ${ }^{6}$ In other words, all measurements done in this experiment match
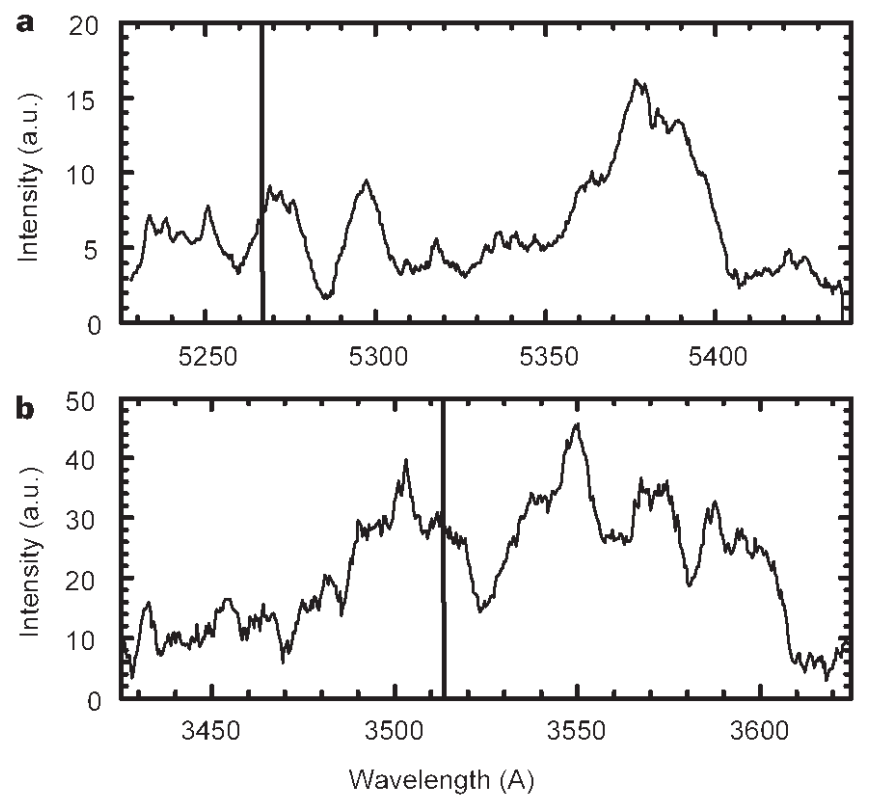

Figure 4 Spectra of harmonics. Shown are spectra of the second (a) and third (b) harmonics at $\theta=90^{\circ}, \varphi=50^{\circ}$ for 0.8-J pulse energy and $6.2 \times 10^{19} \mathrm{~cm}^{-3}$ electron density. Vertical lines indicate the wavelengths of the unshifted second and third harmonics. The intensities are plotted in arbitrary units. The spectra do not change with variation of $\varphi$ at any specific $\theta$, so the angular distributions measured are not affected by the choice of filter bandwidth. qualitatively with the prediction of incoherent nonlinear Thomson scattering of electrons without drift motion; the results appear not to be affected by the existence of plasma waves, probably due to destructive coherent interference. The absolute scattering efficiency was measured to be $8 \times 10^{-4}$ and $1 \times 10^{-4}$ photons per electron per pulse for the second and third harmonics (including both the unshifted and red-shifted spectral components), respectively; these efficiencies were measured at $\theta=90^{\circ}$ and $\varphi=50^{\circ}$, for an angle of collection of $7 \times 10^{-3} \mathrm{sr}$. These numbers match reasonably well with the theoretical predictions for incoherent nonlinear Thomson scattering, which are respectively $8 \times 10^{-4}$ and $5 \times 10^{-4}$ photons per electron per pulse.

\section{References}

1. Thomson, J. J. Conduction of Electricity through Gases (Cambridge Univ. Press, 1906).

2. Vachaspati Harmonics in the scattering of light by free electrons. Phys. Rev. 128, 664-666 (1962).

3. Brown, L. S. \& Kibble, T. W. B. Interaction of intense laser beams with electrons. Phys. Rev. 133, A705-A719 (1964).

4. Sarachik, E. S. \& Schappert, G. T. Classical theory of the scattering of intense laser radiation by free electrons. Phys. Rev. D 1, 2738-2753 (1970).

5. Castillo-Herrera, C. I. \& Johnston, T. W. Incoherent harmonic emission from strong electromagnetic waves in plasmas. IEEE Trans. Plasma Sci. 21, 125-135 (1993).

6. Esarey, E., Ride, S. K., \& Sprangle, P. Nonlinear Thomson scattering of intense laser pulses from beams and plasmas. Phys. Rev. E 48, 3003-3021 (1993).

7. Hartemann, F. V. \& Luhmann, N. C. Jr. Classical electrodynamical derivation of the radiation damping force. Phys. Rev. Lett. 74, 1107 1110 (1995).

8. Hartemann, F. V. High-intensity scattering processes of relativistic electrons in vacuum. Phys. Plasmas 5, 2037-2047 (1998).

9. Maine, P. et al. Generation of ultrahigh peak power pulses by chirped pulse amplification. IEEE J. Quantum Electron. 24, 398-403 (1988).

10. Esarey, E. et al. Nonlinear analysis of relativistic harmonic generation by intense lasers in plasmas. IEEE Trans. Plasma Sci. 21, 95104 (1993).

11. Esarey, E. \& Sprangle, P. Generation of stimulated backscattered harmonic generation from intense-laser interactions with beams and plasmas. Phys. Rev. A 45, 5872-5882 (1992).

12. Meyer, J. \& Zhu, Y. Second harmonic emission from an underdense laser-produced plasma and filamentation. Phys. Fluids 30, 890-895 (1987).

13. Basov, N. G. et al. Investigation of $2 \omega_{0}$-harmonic generation in a laser plasma. Sov. Phys. JETP 49, 1059-1067 (1979).

14. Malka, V. et al. Second harmonic generation and its interaction with relativistic plasma waves driven by forward Raman instability in underdense plasmas. Phys. Plasmas 4, 1127-1131 (1997).

15. Englert, T. J. \& Rinehart, E. A. Second-harmonic photons from the interaction of free electrons with intense laser radiation. Phys. Rev. A 28, 1539-1545 (1983).

16. Bula, C. et al. Observation of nonlinear effects in Compton scattering. Phys. Rev. Lett. 76, 3116-3119 (1996).

17. Brunel, F. Harmonic generation due to plasma effects in a gas undergoing multiphoton ionization in the high-intensity limit. J. Opt. Soc. Am. B 7, 521-526 (1990).

18. Chen, S.-Y. et al. Evolution of a plasma waveguide created during relativistic-ponderomotive self-channeling of an intense laser pulse. Phys. Rev. Lett. 80, 2610-2613 (1998).

19. Le Blanc, S. P. et al. Temporal characterization of a self-modulated laser wakefield. Phys. Rev. Lett. 77, 5381-5384 (1996).

Acknowledgements: We thank G. Mourou, R. Wagner, and X.-F. Wang for discussions. The work of S. C. and A. M. was supported by US NSF and that of D. U. by the Office of Energy Research, US DOE. 